\title{
Perinatal Loss
}

\section{A Critique of Current Hospital Practices}

Irving G. Leon, Ph.D.

Summary: Dramatic improvements in the hospital management of perinatal loss have taken place in the past 20 years. However, there has been no critical examination of current approaches. Four possible hazards of current hospital practice are described: 1) Institutionalization of bereavement. Instead of offering parents an empathic awareness of the unique dimensions of their perinatal loss, caregivers often interact according to detailed behavioral protocols. 2) Idealization of contact with the dead baby: This approach may equate actual physical contact with the dead child with the more complicated and variable process of mourning. 3) Homogenization of grief: Counselors tend to denigrate different grief responses by focusing on a preconceived grief reaction. Thus, they may mistakenly label many such reactions pathologic if they deviate from the rigidly prescribed "norm." 4) Lecturing the bereaved: Telling parents the "right" thing to do may deprive them of a crucial aspect of the process that empowers parents after they experience the helplessness associated with perinatal loss - that of making their own decisions. These problems are illustrated by a clinical vignette, and alternative approaches are explored.

\section{Introduction}

$\mathrm{F}$ ew areas in medical practice have undergone as radical a revision and as dramatic an improvement in so short a time as the professional response to peri-

From the Department of Psychiatry, Child and Adolescent Psychiatric Hospital, The University of Michigan Medical Center, Ann Arbor, Michigan.

Address correspondence to: Irving $G$. Leon, Ph.D., Child and Adolescent Psychiatric Hospital, 1500 E. Medical Drive, Ann Arbor, MI 48109-0390

(313) 662-2055 natal loss. About 20 years ago, medical caregivers typically tried to prevent parents from mourning a stillborn or neonatal death by prohibiting any contact with the dead child, disposing of the body unceremoniously and anonymously, prescribing tranquilizers for the parents to dull any expression of shock or grief, advising them to forget the experience, and often suggesting another pregnancy soon. Today, a completely opposite approach is recommended. Parents are encouraged to see, touch, hold, name, and bury their stillborn or dead infant to make the child's life and death seem more real. They are discouraged from taking tranquilizers; instead, parents are encouraged to share their feelings about the loss with each other. They are also advised not to attempt another pregnancy for six months to a year, the period now considered long enough to mourn their child's death. ${ }^{1-5}$ This dramatic turnaround in medical procedure is illustrated by the contrast between the comments by Kennel et $\mathrm{al}^{6}$ (considered radical in 1970) and, more recently, by Zeanah. ${ }^{7}$ Kennel et al suggested that parental contact with their dead new- 
borns is not associated with pathologic grief, while Zeanah observes that encouraging such contact is now so universally recommended it is probably impossible to measure the value of this practice rigorously by random assignment into contact and noncontact groups. The current "management" of perinatal loss is so taken for granted that it has become an orthodoxy without having been examined critically.

What follows is a critique of current hospital practices for dealing with perinatal loss. The burgeoning literature in this field has been reviewed elsewhere. ${ }^{7,8}$ Unfortunately, no systematic quantitative studies assessing hospital management of perinatal loss have yet been reported. Therefore, this critique draws on qualitative data such as the comments of bereaved parents about their hospital experiences and on various publications that offer professional counselors caregiving tips for managing perinatal loss.

\section{Institutionalization of Bereavement}

Before perinatal death was recognized as a significant loss, its management was easy: nothing needed to be done. Since it was believed that no attachment to the child had yet been established, there was no person to mourn. It has been suggested that the denial by medical caregivers of the significance of perinatal death, as well as of their own feelings of grief, responsibility, guilt, and inadequacy associated with the death, are defense mechanisms. ${ }^{1,4,5,8-13}$ In any case, professional caregivers' lack of emotional involvement with the dead baby was logically consistent with the prevailing view that because no parental bond with the child existed, to encourage parental grieving would be needlessly painful.

Of course, it is now much better understood how powerfully attached most parents become to their unborn children over the course of pregnancy, experiencing them more and more as distinct individuals after quickening and as delivery approaches. ${ }^{14-19}$ Guided by this greater awareness of prenatal parental attachment, medical caregivers now recognize the importance of facilitating the mourning of this loss. Changing the beliefs of these professionals, however, may not completely remove their defenses. No doubt caregivers now are much more sensitive to bereaved parents' grief after perinatal loss than they were in the past. Yet caregivers' responses are often dictated by highly specific behavioral protocols, resulting in a disturbing "institutionalization of bereavement."

Procedures that easily turn into rules may dictate set interactions with the bereaved, giving these rules priority over empathic listening and responsiveness. As Furman $^{20}$ recently observed, "Measures for helping therefore tend to be formally categorized rather than individually attuned to the needs of the particular family." Carr and Knupp ${ }^{21}$ provide a detailed protocol of over 70 instructions for professionals on how they should approach bereaved parents. Hutti"2 similarly offers "a quick reference table" of over 40 predesigned recommendations caregivers can offer parents to help them cope with perinatal loss. When such specific guidelines govern natural interactions, parents may feel their experience has been so regimented they are unable to regain mastery over their lives, whereas the true aim of the caregiver is just the opposite - to foster parents' sense of control.
Bereaved parents have complained of feeling they were expected to cry openly in the presence of medical caregivers whom they hardly knew, as if their expressions of grief were meant to fulfill the caregivers' agendas rather than meet their own needs.

It is not so much the sensitivity of individual recommendations that is being challenged here as it is the total impact of such a protocol on a practitioner - the implication that he or she must be taught how to respond humanely and sensitively to another person - what to do, what to say, and, ultimately, what to feel. In reality, sensitive, effective caregivers incorporate many, but not all, of these suggestions naturally, accommodating them to the specific needs of a family. A protocol cannot generate empathy if the practitioner lacks the capacity for it. Precise instructions may provide a refuge for caregivers who use rules to avoid genuine emotional involvement with bereaved parents. Conscientious professionals who sincerely want to do "the right thing" may derive comfort from such rigid rules, and perhaps such an approach, even if emotionally hollow, is a significant improvement over the absence of any demonstration of concern. However, professionals who blandly recite an explanation of the stages of grief may be erecting a defense mechanism against their own sadness just as they had earlier in their overt denial of the loss. Learning the right words does not assure singing in harmony. Some parents complain that their caregivers seemed to play at being emotional, that they seemed "phony," mouthing prepared lines rather than responding spontaneously and empathetically to the parents' sense of loss.

Meaningful alternatives to extensive protocols are available to 
aid professional intervention after perinatal loss. It is possible to provide direction and guidance for caregivers without becoming restrictively specific. Based on her phenomenological study of 20 women who miscarried, SwansonKauffman ${ }^{23}$ has delineated five aspects of caring. Fundamental to caring is to understand the personal meanings of the loss; to resonate emotionally with the mother's feelings; to offer her realistic support, nurturance, and protection; to facilitate the expression of her grief; and to help maintain her faith in her capacity to come through her loss a functioning, whole person. While SwansonKaufmann's model for caring is designed for the nursing profession, her categories correspond closely with a description of short-term psychodynamic psychotherapy for mothers who have suffered perinatal loss. ${ }^{8}$ By seeing that the same principles may apply to both medical and psychotherapeutic practice, we can breach the schism between these different forms of caregiving. Providing a flexible alternative to specific instructions, Swanson-Kauffman ${ }^{23}$ concludes, "The categories are offered as insights, not formulas. They are descriptions and interpretations not prescriptions - and should be used accordingly." She stresses the quality of the relationship between caregiver and receiver rather than procedures. This emphasis allows a much more individualized and responsive approach to caregiving than can be achieved with a behavioral protocol.

\section{Idealization of Contact with the Dead Baby}

The cornerstone of current hospital practice in managing perinatal loss is to encourage contact with the dead baby both directly (by viewing, touching, and holding) and indirectly (through photographs, fingerprints and footprints, and memorabilia associated with the child). Virtually all current researchers and clinicians have endorsed this kind of intervention and the many useful functions it serves: Denial of the loss is reduced by making both the pregnancy and the death more real; mourning the dead child is facilitated by visual reminders over which the parents can grieve, giving them one of their few opportunities to express their love for their child concretely; their diminished self-esteem may be repaired by allowing them to identify proudly with cherished aspects of themselves that they see in their creation. Constructing a concrete identity for the dead child by naming him or her, as well as conducting a funeral service for, and establishing memories of, the child are important steps in the process in light of society's continuing (albeit lessening) unwillingness to acknowledge the dead infant as a real person..$^{2,2428}$

The current climate of acceptance that allows parents to choose whether to have contact with their dead child is a testimony to the courage, sensitivity, and humanity of such medical pioneers as Kennell and Klaus, ${ }^{1,6,29}$ Lewis, ${ }^{3,30}$ and Kellner et al, ${ }^{31}$ who challenged the standard hospital practice by which this parental right was discouraged or prohibited. That even now not all hospitals offer this contact indicates the need for further education about perinatal loss and the value of promoting such attitudes.

However, the overt behavior of viewing and holding one's dead child must be clearly distinguished from the much more complicated internal process of mourning - the process of separating oneself from the relationship with the deceased. While physical contact with the dead child is often a valuable outlet for the expression of grief, it is not the only one, nor is it necessarily optimal for all parents.

We may be in danger of repeating misunderstandings concerning ways of easing the mourning process after perinatal loss which occurred not long age in promoting parent-infant attachment. Because Klaus and Kennell's ${ }^{29}$ concept of bonding was simplistically and exclusively equated with early parent-infant interaction, this concept was challenged in reviews of studies that questioned the enduring effects of early interaction. . $^{32-34}$ Unrealistically high expectations for a simple action ensuring as complicated a process as attachment or mourning must be tempered. Otherwise, the inevitable disappointment which follows will begin to undo the real progress that has been accomplished in developing more humane hospital practices in fostering the growth or mourning of attachments at birth.

In fact, there is virtually no quantitative evidence to support the belief that parental contact with the dead child facilitates recovery from perinatal loss. On the contrary, several studies have found that parental contact had no significant effect on the course or outcome of mourning ${ }^{35-37}$ While Murray and Callan ${ }^{38}$ report that the degree of parents' satisfaction about the time they had spent with their dead child was directly related to their feelings of self-esteem two years after their loss, these authors suggest there is "the need to consider parents' level of satisfaction with the type of contact, as well as ... how often they saw, touched, or held their infant." One clinical study $y^{8}$ found that con- 
tact with the dead child did not determine the course of mourning. Cooper's ${ }^{39}$ report of 17 British couples recovering from the trauma of stillbirth is rarely reported. Although none of the children cited in this report were named or buried and few of the couples chose to view their stillborns, the trajectory of their grief appeared quite similar to that of couples who had had contact with, named, and buried their stillborn children.

We should question the growing conviction that such contact is a simple panacea and try instead to understand why this contact is beneficial. For some parents, interacting with their dead child may be less meaningful and memorable than the professional caregiver's emotional resonance with their feelings. As Furman ${ }^{20}$ notes, "The newly introduced policy of showing the dead baby to the parents and involving them in the funeral arrangements is often pursued as an end in itself rather than as a part of an empathic professional relationship." When the goal is to "show the baby" and not to listen to, understand, and respond sensitively to parents as individuals, such contact may lose much of its value. The timing and manner of offering contact should flow naturally from the interaction between the caregiver and the parents, rather than being part of a predetermined protocol. If caregivers direct their energy solely toward determining the "right way" to offer contact as part of a protocol for all parents, they will lose sight of the importance of accommodating to different parental reactions and needs in an empathic relationship.

For many parents, the act of being offered a choice may be crucial. Such a gesture may mitigate the debilitating sense of helpless- ness engendered by perinatal loss by legitimating and supporting the parents' right to decide what is right for themselves, rather than being told or persuaded what to do. The earlier policy of forbidding parental contact was misguided not only because it prevented many of the productive results of such contact but also because it denied parental authority and autonomy by usurping this important decision. However, the natural benefits of such contact may be diminished if it is achieved by sacrificing the importance of empowering parents.

The parents must not be rushed through the painful process of digesting the news of their baby's death, considering the possibility of viewing their dead child, and assimilating information of how parents usually respond to this experience. According to Lewis and Bourne' $\mathrm{s}^{40}$ guide for medical caregivers who deal with perinatal loss, "The baby should not be bundled away, although there may now be some danger of staff rushing in with a new dogma which demands that every parent obediently inspects and holds every stillbirth immediately. A different danger is that such procedures will become institutionalized, rigid, and stripped of meaning. Tact and patience are important ... but unfortunately there is often an atmosphere of anxious noise and rush in the delivery room so that people do not have the space to think."

Parents who decide not to view their dead baby are not necessarily in danger of unresolved grief. Many women find alternative, personal ways to memorialize and identify their stillborn children in artistic creations without actually having seen them. ${ }^{8}$ Just as mothers form bonds with their babies regardless of whether or not there has been early interaction with them, mothers can mourn their dead children even if they choose not to view them. Of course, in the case of perinatal death, there will be no other opportunities for contact, making this decision permanent. Klaus and Kenne $1^{1,41}$ denounced the burden of guilt which can be placed on mothers who, not having "bonded" with their neonates, can be told to feel something irrevocable had been lost. Since there is no empirical evidence to indicate that the parents who choose not to view their dead child are less likely to recover from this loss, we must not foster a similar burden of guilt in these parents.

\section{Homogenization of Grief}

Bereaved parents should be told the recognized patterns of grief so they understand that temporary depressive feelings, such as lethargy and sadness, and/or somatic reactions, such as eating or sleep disorders, are normal and not pathologic responses to such a loss. This knowledge will relieve parents who may otherwise feel that their intense, debilitating reactions after a perinatal death are signs of emotional disturbance. Also, by understanding the range of normal bereavement responses, parents are then able to recognize the signs of unresolved grief, whereby the grieving process is arrested at a particular phase (i.e., inhibited or chronic grief) or else takes a pathologically variant form (i.e., hypochondriasis). ${ }^{4246}$ The bereaved parents can then seek appropriate counseling or psychotherapy. ${ }^{45,47-48}$

Drawing up such a blueprint of "normal" grief responses carries its own danger, however: It may restrict the range of normative expressions of mourning unreasonably. The bereaved family may 
begin to feel there is only one "right" way to mourn and that their expressions of grief are "wrong." In a study of bereaved parents, Miles and Demi ${ }^{49}$ discovered that parents who perceived themselves as grieving incorrectly or behaving inappropriately suffered from "grief guilt" to a degree far exceeding that felt in survivor guilt ("I should have died too/instead"), recovery guilt (felt when emerging from grieving), and moral guilt (the belief that a child's death was a punishment for some wrongdoing by the parent[s]). Narrowly limiting the ways in which parents may experience grief results in excluding alternative responses, thus defeating the value of normalizing grief reactions.

Conceptualizations of, and interventions for, perinatal loss appear to be especially vulnerable to this "homogenization of grief," the tendency to minimize the different ways people respond to grief by focusing on a modal grief reaction. Although some researchers ${ }^{8,50} \mathrm{em}^{-}$ phasize considerable variation in individual responses, most describe bereavement reaction as a typical sequence of stages. It has been assumed that the course of grief is much the same for all bereaved parents, perhaps because there has been no extended relationship with the baby as a separate person. While it is axiomatic for grief counselors to inquire about the relationship of the bereaved to a deceased child or adult, it is atypical for perinatal-loss specialists to investigate the unique meanings which the pregnancy and death have for both parents. It is a sad paradox that caregivers' increased awareness that parents feel perinatal death as the real, distinct loss of another person is complemented by their disinterest in the uniqueness of the prenatal bond. Rarely do perinatal-loss counselors obtain an adequate history of the bereaved parents that includes an appreciation of earlier losses, if any, and of such things as their problems, relationships, and coping skills. While it is unrealistic to expect to construct such a background at the time of the death, the value of follow-up contact with the grieving parents may depend in part on how sensitively a counselor can recognize the different dimensions the loss has for each parent.

A professional caregiver can supply this understanding only in the context of the highly personal experience of a perinatal loss. The ability of a caregiver to provide parents with accurate information about their perinatal loss will depend on the professional's broad knowledge of parental reactions, refined and modified by an appre ciation of what the pregnancy and the child meant to those particular parents. A counselor can best help parents who have experienced perinatal loss by taking the time and effort to get to know them. In spite of the realistic limits on their time, even the busiest medical caregivers will find the time if they appreciate the importance of their relationship with the bereaved parents who consult them. Unfortunately, in both the clinical and the research literature, the circumstances of loss and the sociodemographics of bereaved parents are studied more intensively and granted a greater causal role in determining outcome than are the personalities of the couple.

The assumption that pregnancy loss inevitably and solely involves mourning the death of one's baby is open to question. ${ }^{51}$ Such a loss may have other important psychological and emotional effects; it may frustrate the couple's wish to parent, it may revive earlier emo- tional conflicts, and it may be a powerful blow to the parents' selfesteem. ${ }^{8,51}$ Although a psychotherapist may be best equipped professionally to understand and sort out these different emotions and their impact, any sensitive listener, if unencumbered by a mindset that recognizes only one interpretation of these losses, can also perceive such differences and appreciate the uniqueness of the loss to the parents.

Many studies are all too ready to classify intense grief after perinatal loss as pathologic or deviant behavior, ${ }^{35,36,52-54}$ instead of understanding it as just one of a broad range of normal responses. This tendency suggests how deeply ambivalent mental-health investigators are about the intensity of the emotions expressed during grieving; they readily accept such reactions as normal in their clinical work but all too easily label the same feelings pathologic unresolved grief when they appear in research subjects. Perhaps this attitude reflects our society's discomfort with, and intolerance of, the intense feelings of grief. The factor-analytic longitudinal studies by Toedter and Las$\operatorname{ker}^{55,56}$ clearly demonstrate that the initial intensity of grief, as expressed by crying and feelings of sadness, is less useful in predicting the resolution of the loss than other independent factors such as despair and difficulty in coping. Lewis ${ }^{30}$ describes a newly bereaved mother trying to "walk" her dead baby and frantically kissing his navel, mouth, and penis and then explains this seemingly mad behavior not as deviant but as one acceptable way of expressing and dealing with profound grief. Lewis obviously understands the importance of attempting to understand the meaning of a parent's behavior and feelings after perinatal loss, no matter how pathologic they may 
appear, and then considering whether such reactions promote or interfere with the process of mourning.

\section{Lecturing the Bereaved}

Following unexpected perinatal loss, shocked parents will often turn to medical caregivers for help in structuring the cognitive, emotional, and behavioral disorganization they are experiencing. Important decisions about whether to have contact with the dead baby and what should be done with the body must be made as the parents struggle with intense, often mixed, emotions as well as diminished capacity in memory, attention, concentration, and judgment. A sensitive caregiver will offer the parents realistic decision options, clarify their mixed feelings, and serve as an "auxiliary ego" to foster in them a more accurate understanding of their sad experience. By preparing the bereaved parents for the range and intensity of the grief they may experience, the caregiver reduces the likelihood of further trauma and allays the fear parents often have of "going crazy." The caregiver's role in this kind of crisis intervention is active and direct: he or she will make concrete suggestions, such as urging the parents to share feelings with one another and explaining clearly to surviving children the reasons for the new baby's death. ${ }^{1}$

The parents' overwhelming sense of helplessness, inability to function normally, and poignant appeal for help may fuel an irresistible urge in the caregiver to respond paternalistically. With benevolent motives, the caregiver may begin to dictate to the bereaved what they "should be" doing and feeling. It is difficult to give sound advice based on the caregiver's clinical experience of what has helped other parents without intruding on parental autonomy and ignoring individual differences. It is necessary to listen, attempt to understand, and respond compassionately to parents' suffering, rather than to assume it can be made to go away by authoritative directives from the caregiver.

Caregivers usually try to convince the bereaved parents that they are not to blame for their perinatal loss. When parents misunderstand the cause of death because of cognitive confusion or misinformation, it is necessary to correct such inaccuracies. However, in a population that is relatively sophisticated and well-educated, the mother usually knows intellectually she did not cause the death but feels irrationally, though strongly, that she is to blame. Although a caregiver, wishing to relieve parents' sense of guilt, may try to persuade them they are not to blame for their baby's death, such an approach may unempathically disregard the parents' experience. It may be more helpful to acknowledge the mother's self-blame and point out, perhaps, that she is being very hard on herself.

Parents are usually advised to wait at least six months after suffering a perinatal loss before attempting another pregnancy. This suggestion is based on the hypothesis that it is first necessary to mourn the lost baby before a separate attachment can be formed to a new child. The well-meaning intention is to avoid the possibility that the loss will remain unresolved and dominate the life and identity of the "replacement" baby. $8,57-58$ However, there may be overriding psychological reasons for pursuing a subsequent pregnancy after the perinatal loss. The couple's wish to parent may become intense, especially if their advancing age makes waiting psychologically stressful as well as biologically unwise. The parents' need to repair their damaged self-esteem through a successful pregnancy may become pronounced, especially after an early-pregnancy loss, which may be experienced as the loss of a part of oneself..$^{8,59-61}$ Murray and Callan ${ }^{38}$ report that a successful pregnancy after a perinatal loss is among the most important factors associated with parents experiencing less depression and a greater sense of well-being within the next two years. Theut ${ }^{53}$ and her colleagues also find that resolution of perinatal loss is often achieved following the next successful delivery.

The caregiver may exacerbate the mother's loss of self-esteem, the result of her sense of helplessness in being unable to save her baby, by deciding the timing of her next pregnancy for her. She may feel her own control over, and pride in, her reproductive destiny threatened by what she perceives as the caregiver's authoritative, albeit well-meaning, usurping of her decision-making. In the only quantitative study that examined pregnancy planning (among 24 women) following perinatal loss, it was found that of the 14 women who were advised to wait at least six months before attempting another pregnancy, only four followed this advice; 17 (almost $75 \%$ ) chose to wait less than six months. ${ }^{62}$ Not only was advising them to wait clearly ineffective; they were significantly more unhappy when so advised than were women given no advice at all. Many of their reasons for not waiting echo those discussed above: the need to alleviate the crushing blow to their self-esteem; the intolerable sense of emptiness, feelings of failure, and fear of infertility; as well as the developmental wishes to 
parent - the need to get on with life and the fear of advancing age. The authors conclude that "because of the many personal needs and unique circumstances each mother considers in deciding when to attempt another pregnancy, the timing of subsequent pregnancy is a personal decision." ${ }^{62}$ They recommend that medical caregivers adopt an educational, informative role rather than an advising, prescriptive one. The possibility of medical complications in another pregnancy begun too soon after a pregnancy loss must also be discussed with the parents, especially if testing or other medical interventions are necessary to increase the likelihood of future reproductive success.

These concepts are illustrated below in the description of how a perinatal loss was handled in a large hospital which, several months earlier, had sponsored an inten sive three-day workshop on managing perinatal loss for obstetric nurses and perinatalloss specialists. Minor details of the circumstances of the loss were changed in order to preserve confidentiality.

\section{A Clinical Vignette}

A woman learned that the fetus she was carrying at less than four months' gestation was fatally handicapped and could not survive. The pregnancy was terminated. Both parents were repeatedly encouraged by the perinatal-loss counselors to view and hold the fetus. They refused emphatically, explaining that the fetus did not seem like a baby to them. The counselors pressed them to name the fetus and learn its sex, but again they refused, giving the same reason. When asked to provide a name, they finally offered "Fuzzy," a nickname coined by their 3-year-old child during the pregnancy. One counselor would not use this name, writing down "Baby" instead. Another photographed the fetus and gave the roll of film to the parents (who felt they would never want to develop it). Finally, a Polaroid snapshot was taken of the fetus amid ornaments in a basket. The photo was placed on a nearby dresser and the parents were told they could look at it "when you're ready." The parents found this entire experience "bizarre," especially the snapshot of a tiny fetus surrounded by ornaments bigger than itself, one blue (if it was a boy) and one pink (in case it was a girl). Yet they both felt the overall nursing care the mother received (unconnected with the perinatal-loss specialists) was excellent; the mother exclaimed that she "was treated like a queen."

In this case, the perinatal-loss counseling was clearly dictated by a prior protocol, the institutionalization of bereavement mentioned earlier, which ignored the way the parents experienced their loss. Instead of responding to the parents' feeling that this was the end of a pregnancy rather than the loss of a new life, the counselors rigidly assumed that these parents, in fact all parents, must mourn the death of a baby even when the parents do not consider the fetus a baby to be mourned, a good example also of the homogenization of grief. Thus the counselors stubbornly encouraged the parents to look at and name the fetus, believing this would facilitate mourning (an instance of idealization of contact with the dead baby) and ultimately ignored the parents' request not to see the fetus by leaving a snapshot behind. The less-than-subtle pressure to look at the snapshot ("when you're ready") seemed condescending, dictating that this was "the right thing to do" (they were lecturing the bereaved). By their actions and insistence on following a set protocol for grieving the counselors may very well have added to the burden of parental guilt by suggesting the parents did not know what was best for themselves. The enactment of each of these four concepts demonstrates a repeated lack of empathy with these parents' experience.

Other problems in caregiving behavior are evident in this example. The strange ("bizarre," to the mother) nature of the picture of the tiny fetus surrounded by ornaments may have increased the parents' sense of disorientation, in itself a common reaction following perinatal loss. Also, by refusing to accept the name provided by the sibling, the caregivers interfered with the parents' attempt to treat the death of the fetus as a family loss in which the sibling, though absent, could participate. While the caregivers' efforts were no doubt well-intentioned, they were misguided and insensitive to the parents' feelings. The nurturing care and concern the mother received from the nursing staff gave both parents the support they needed, in sharp contrast to their treatment by the perinatal-loss counselors, who seemed to be concerned mainly with eliciting "correct" grief reactions. The mother's delight in being "treated like a queen" - like royalty - by the nurses may have ameliorated her loss of self-worth. The final lesson of this history is that parents often truly know what is best for themselves and that we, as caregivers, must become better listeners and let our patients teach us when they tell us what they need.

\section{Conclusions}

Each of the four aspects of hos- 
pital management of perinatal loss criticized here is rooted in wellmeaning attempts to improve the overall quality of patient care. Protocols were designed to promote a consistent approach to bereaved parents rather than leave such an important responsibility up to the intuitive reactions of individual caregivers who, in the past, commonly avoided dealing with their own and patients' feelings about these losses. The emphasis on parents viewing, touching, and holding their dead babies was meant to correct past institutional prohibitions against these fundamental parental rights. It was based on clinical experience of how effective such contact can be for many parents in helping them make sense of what and who they have lost. Caregivers and parents now should understand that there are patterns of grief and that thoughts, feelings, and behavior which would once have been considered pathologic and would be discouraged are in fact normal. Providing guidance for parents whose thinking may be clouded and judgment impaired at a time of upheaval is a natural human response and a cornerstone in the active approach to crisis management.

The problem is not with the underlying intent of each of these practices but that they are implemented in ways that do not allow recognition of the normal diversity of grief reactions, so that caregivers are unable to offer help geared to the individual needs of the bereaved. Whether by directing caregiver behaviors, magnifying the importance of physical contact with the dead baby, universalizing a single bereavement reaction, or telling parents what to do and how to feel, all perinatal loss is reduced to a single, oversimplified event. It is assumed that perinatal loss is more or less the same for all parents who undergo it and that they can all be helped by much the same approach. Since there are common elements in parental responses to perinatal loss, a consistent approach, even if relatively rigid, will show some sensitivity to parental feelings. However, empathy is most healing when it is free of the rigid restraints of caregiver agendas. As Furman ${ }^{61}$ has described, empathic resonance with bereaved parents is psychologically difficult to achieve and emotionally exhausting: "Even after many years of professional experience in this area, I still find it difficult personally to work with parents in this situation because in order to really help, one has to involve oneself totally anew with each individual who goes through it. When giving oneself, one must strive to find a golden middle road of being able to feel and yet not totally identify with them." Caregivers who frequently confront this loss, such as those who work in neonatal intensive-care units, may need to limit the number of bereaved families for whom they are responsible in order to avoid "burn-out."

The emotional rewards for the caregiver who is allowed to share in this most private grief can be profound. He or she participates in a vital way in the healing process, not with a flurry of activity but by listening unhurriedly, understanding the meaning of the loss to the bereaved family, and helping the parents express their painful and conflicting feelings in words. Bereaved parents never forget the understanding, respect, and genuine warmth they received from caregivers, which can become as lasting and important as any other memories of their lost pregnancy or their baby's brief life.

\section{REFERENCES}

1. Klaus M, Kennell J. Parent-Infant Bond ing. 2nd ed. St Louis: CV Mosby; 1982.

2. Peppers L, Knapp R. Motherhood and Mourning: Perinatal Death. New York: Praeger; 1980.

3. Lewis E. The management of stillbirth: coping with an unreality. Lancet. 1976;2:619-620.

4. Cullberg J. Mental reactions of women to perinatal death. In: Morris $\mathrm{N}$, ed. Psychosomatic Medicine in Obstetrics and Gynaecology. Basel: Karger; 1972:326329.

5. Giles PF. Reactions of women to perinatal death. Aust N Z J Obstel Gynaecol. 1970;10:207-210.

6. Kennell J, Slyter H, Klaus M. The mourning response of parents to the death of a newborn infant. NEngl JMed. 1970;283:344-349.

7. Zeanah C.Adaptation following perinatal loss: A critical review. JAm Acad Child Adolesc Psychiatry. 1989;28:467-480.

8. Leon 1. When a Baby Dies/Psychotherapy for Pregnancy and Newborn Loss. New Haven, CT: Yale University Press; 1990.

9. StackJ. Reproductive casualties. Perinat Press. 1982;6:31-36.

10. Kowalski K. Managing perinatal loss. Clin Obstet Gynecol. 1980;23:1113-1123.

11. Knapp R, Peppers L. Doctor-patient relationships in fetal/infant death encounters. J Med Educ. 1979;54:775-780.

12. Bourne S. Stillbirth, grief, and medical education. BMJ. 1977;1:1157.

13. Bourne $S$. The psychological effects of stillbirths on women and their doctors. $J R$ Coll Gen Pract. 1968;16:103-112.

14. Zeanah C, Keener M, Anders T. Developing perceptions of temperament and their relation to mother and infant behavior. I Child Psychol Psychiatry. 1986;27:499-512.

15. Condon J. The parental-foetal relationship - a comparison of male and female expectant parents. I Psychosom Obstet Gynecol. 1985;4:271-284.

16. Zeanah C, Keener M, Stewart L, Anders T. Prenatal perception of infant personality: a preliminary investigation. $J \mathrm{Am}$ Acad Child Psychiatry. 1985;24:204-210.

17. Weaver RH, Cranley M. An exploration of paternal-fetal attachment. Nurs Res. 1983;32:68-72.

18. Lumley J. Attitudes to the fetus among primigravidae. Aust Paediatr $J$. 1982;18;106-109.

19. Cranley M. Development of a tool for the measurement of maternal attachment during pregnancy. Nurs Res. 1981;30:281-284. 
20. Furman E. Foreword. In: Leon I. When a Baby Dies/Psychotherapy for Pregnancy and Newborn Loss. New Haven, CT: Yale University Press. 1990:xi-xii.

21. Carr D, Knupp S. Grief and perinatal loss/a community hospital approach to support. J Obstet Gynecol Neonatal Nurs. 1985;14:130-139.

22. Hutti M. A quick reference table of interventions to assist families to cope with pregnancy loss or neonatal death. Birth. 1988;15:33-35.

23. Swanson-Kauffman $K$. Caring in the instance of unexpected early pregnancy loss. Top Clin Nurs. 1986;8:37-46.

24. Lovell A. Some questions of identity: late miscarriage, stillbirth and perinatal loss. Soc Sci Med. 1983;17:755-761.

25. Cohen L, Zilkha S, Middleton J, O'Donnohue N. Perinatal mortality: assisting parental affirmation. Am J Orthopsychiatry. 1978;48:727-731.

26. Helmrath $\mathrm{T}$, Steinitz E. Death of an infant: parental grieving and the failure of social support. I Fam Pract. 1978;6:785-790.

27. Davidson G. Death of the wished-for child: a case study. Death Educ. 1977;1:265-275.

28. Hagan J. Infant death: nursing interaction and intervention with grieving families. Nurs Fonum. 1974;13:371-385.

29. Klaus M, Kennell J. Mother-Infant Bonding. St. Louis: CV Mosby; 1976.

30. Lewis E. Mourning by the family after a stillbirth or neonatal death. Arch Dis Child. 1979;54:303-306.

31. Kellner K, Kirkley-Best E, Chesborough $S$, et al. Perinatal mortality counseling program for families who experience a stillbirth. Death Educ. 1981;5:29-35.

32. Myers B. Mother-infant bonding: the status of this critical period hypothesis. Dev Rev. 1984;4:240-274.

33. Chess S, Thomas A. Infant bonding: mystique and reality. Am J Orthopsychiatry. 1982;52:213-222.
34. Lamb M. Early contact and maternal-infant bonding: one decade later. Pediatrics. 1982;70:763-768.

35. Nicol M, Tompkins J, Campbell N, Syme G. Maternal grieving response after perinatal death. Med $J$ Aust. 1986;144:287-289.

36. Tudehope D, Iredell J, Rodgers D, Gunn A. Neonatal death: grieving families. MedJ Aust. 1986; 144:290-292.

37. Benfield DG, Leib S, Vollman J. Grief response of parents to neonatal death and parent participation in deciding care. Pediatrics. 1978;62;171-177.

38. Murray J, Callan V. Predicting adjustment to perinatal death. BrJ Med Psychol. 1988;61:237-244.

39. Cooper J. Parental reactions to stillbirth. BrJ Soc Work 1985; 10:55-69.

40. Lewis E, Bourne S. Perinatal death. Bailliere's Clin Obst Gynecol. 1989;3:935953.

41. Kennell J, Klaus M. Mother-infant bonding: weighing the evidence. Dev Rev. 1984;4:275-282.

42. Osterweis M, Solomon F, Green M, eds. Bereavement: Reactions, Consequences and Care. Washington DC: National Academy Press; 1984.

43. Lindemann E. Symptomatology and management of acute grief. Am JPsychiatry. 1944;101:141-148.

44. Parkes CM. Bereavement. Br J Psychiatry. 1985;146:11-17.

45. Raphael B. The Anatomy of Bereavement. New York: Basic Books; 1983.

46. Bowlby J. Attachment and Loss: Loss, vol III. New York: Books; 1980.

47. Volkan V. Psychotherapy of complicated mourning. In: Volkan V, ed. Depressive States and Their Treatment. Northvale, NJ: Aronson; 1985:271-295.

48. Worden J. Grief Counseling and GriefTherapy. New York: Springer; 1982.

49. Miles M, Demi A. Toward the development of a theory of bereavement guilt: sources of guilt in bereaved parents.
Omega. 1984;14:299-314.

50. Outerbridge E, Chance $G$, Beaudry $M$, et al. Support of parents experiencing perinatal loss. Can Med Assoc J. 1983;129:335-339.

51. Leon 1 . The psychoanalytic conceptualization of perinatal loss: a multi-dimensional model. Am J Psychiatry. In press.

52. Theut S, Pedersen F, Zaslow M, et al. Perinatal loss and parental bereavement. AmJPsychiatry. 1989;146:635-639.

53. Theut S, Zaslow M, Rabinovich B, etal. Resolution of parental bereavement after a perinatal loss. J Am Acad Child Adolesc Psychiatry. 1990;29:521-525.

54. LaRoche C, Lalinec-Michaud M, Engelsmann F, et al. Grief reactions to perinatal death: an exploratory study. Psychosomatics. 1982;23:510-518.

55. Toedter L, Lasker J, Alhadeff J. The perinatal grief scale: development and initial validation. Am J Orthopsychiatry. 1988;58:435-449.

56. Lasker J, Toedter L. Acute versus chronic grief: the case of pregnancy loss. Am J Orthopsychiatry. 1991;61:510-522.

57. Lewis E, Page A. Failure to mourn a stillbirth: an overlooked catastrophe. $B r$ J Med Psychol. 1978;51:237-241.

58. Legg C, Sherick I. The replacement child - a developmental tragedy. Child Psychiatry Hum Dex. 1976;7:113-125.

59. StackJ. The psychodynamics of spontaneous abortion. Am J Orthopsychiatry. 1984;54:162-167.

60. Grubb C. Body image concerns of a multipara in the situation of intrauterine fetal death. Matern Child Nurs J. 1976:5:93-116.

61. Furman E. The death of the newborm: care of the parents. Birth Fam J. 1978;5:214-218.

62. Davis D, Stewart M, Harmon R. Postponing pregnancy after perinatal death: perspectives on doctor advice. $J$ Am Acad Child Adolesc Psychiatry. 1989;28:481-487. 